\title{
Accuracy of the typical computed tomographic appearances of fibrosing alveolitis
}

Kenneth T Tung, Athol U Wells, Michael B Rubens, Jackie M E Kirk, Roland M du Bois, David M Hansell

\begin{abstract}
Background-Open lung biopsy is often performed to confirm the diagnosis in patients with suspected fibrosing alveolitis. The superior sensitivity and specificity of high resolution computed tomography (CT) over chest radiography in various diffuse lung diseases suggest that the characteristic appearance of fibrosing alveolitis on high resolution CT might render biopsy confirmation unnecessary.
\end{abstract}

Methods-The chest radiographs and high resolution CT scans of 86 patients (41 with fibrosing alveolitis and 45 with various other diffuse lung diseases) were examined individually and independently by two observers. No clinical information was given and the observers gave a level of confidence when the diagnosis was thought to be fibrosing alveolitis.

Results-The observers correctly and confidently discriminated between fibrosing alveolitis and other diffuse lung diseases on high resolution CT with an accuracy of $88 \%$ and on chest radiography with an accuracy of $76 \%$. The false negative rate for fibrosing alveolitis diminished from $29 \%$ on chest radiography to $11 \%$ on high resolution CT. The false positive rate on chest radiography was $19 \%$ and on high resolution CT $13 \%$; the false positive diagnoses on CT were the result of a few conditions (extrinsic allergic alveolitis, sarcoidosis, cryptogenic organising pneumonia, and pulmonary eosinophilia) which mimicked some of the CT features of fibrosing alveolitis. The superficial similarity of the CT patterns of these conditions are discussed.

Conclusions-High resolution CT is superior to chest radiography in establishing the diagnosis of fibrosing alveolitis and the typical CT appearances are virtually pathognomonic. The diagnostic advantages of CT over chest radiography should further reduce the need for open lung biopsy in this condition.

(Thorax 1993;48:334-338)

The diagnosis of fibrosing alveolitis has traditionally relied on a combination of clini- cal features, a restrictive pattern on lung function tests, and chest radiography showing predominantly lower zone shadowing. ${ }^{2}$ Patients with clinical and radiographic features suggestive of fibrosing alveolitis often have an open lung biopsy performed to confirm or refute the diagnosis. Lung biopsy is considered the gold standard in providing pathological verification of the diagnosis and a guide to prognosis. ${ }^{2-4}$

The radiographic features of fibrosing alveolitis are well known with the typical appearances of reticular shadowing concentrated in the lower lobes. ${ }^{56}$ The chest radiograph in patients with early disease may be normal $^{7}$ and in the later stages the radiographic appearances are often non-specific.

More recently the features of fibrosing alveolitis have been characterised by high resolution computed tomography (CT) and many reports have suggested that the distribution of the disease is virtually pathognomonic for this condition. ${ }^{8-10}$ The CT features of fibrosing alveolitis include ground glass opacification (corresponding to inflammation histologically) in a subpleural crescentic pattern initially confined to the bases and a peripheral fine reticular pattern (denoting established fibrosis) (fig 1). The specificity of the CT appearance of fibrosing alveolitis has not been formally tested. The purpose of this study was to compare the accuracy of conventional chest radiography and high resolution CT in discriminating between fibrosing alveolitis and other diffuse lung diseases.

\section{Methods}

\section{PATIENTS}

Eighty six patients with various diffuse lung diseases who were attending the Royal Brompton National Heart and Lung Hospital and had concurrent chest radiographs and CT scans were included in the study. Patients being investigated for asbestos related lung disease or in whom the final diagnosis was equivocal were excluded. The details of the method of diagnosis and subsequent clinical course were obtained from the case notes (table 1). A histological diagnosis was established by open lung biopsy $(n=49)$, transbronchial biopsy $(n=8)$, or percutaneous needle biopsy $(n=2)$. In the remaining 27 patients the diagnoses which were based on history, clinical examination, serology, radiol-

Received 3 June 1992 Returned to autho 


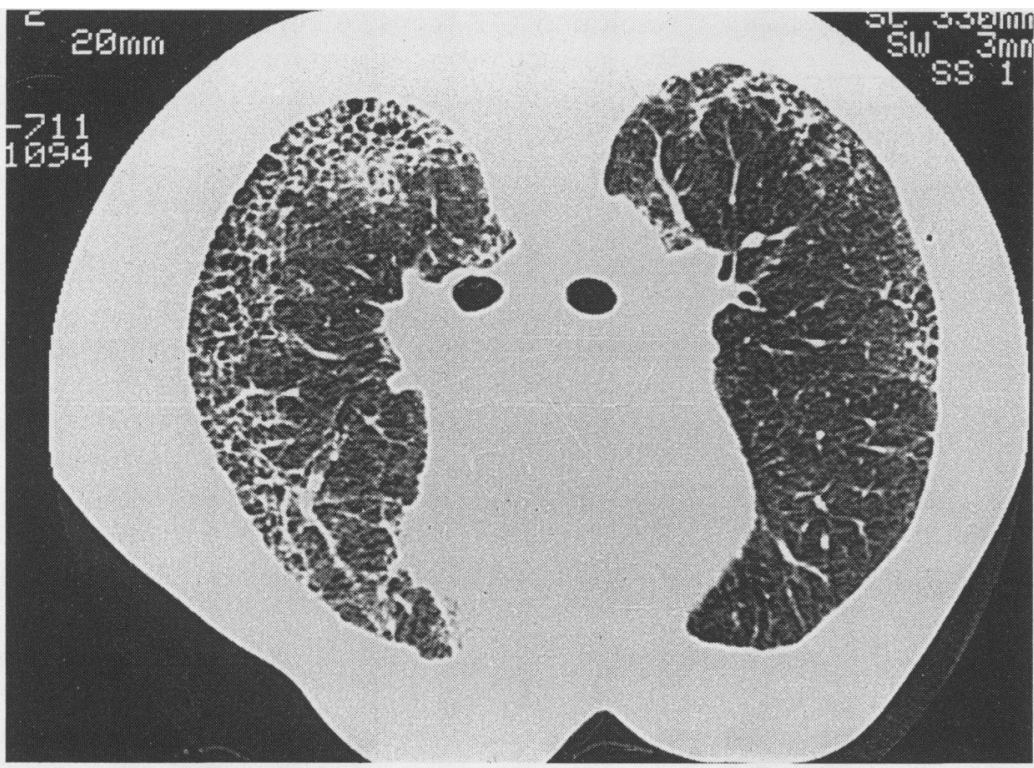

Figure 1 High resolution computed tomography through the lower lobes showing the typical subpleural reticular pattern of established fibrosing alveolitis (patient prone).

ogy, and pulmonary function test evidence were considered definite and were not altered during subsequent follow up. In all 86 patients a definite and specific diagnosis of diffuse lung disease was made. These comprised 41 patients with fibrosing alveolitis and 45 patients with a wide variety of diffuse lung diseases (extrinsic allergic alveolitis, eight; sarcoidosis, seven; cryptogenic organising pneumonia, five; eosinophilic granuloma, five; other, 20).

\section{IMAGING}

Chest radiographs were taken at high kilovoltage $(140 \mathrm{kVp})$ by an air gap technique or taken with an AMBER chest unit also at 140 $\mathrm{kVp}$. Thin section CT scans were performed with an Elscint 2002 machine (Elscint, Haifa,

Table 1 Diagnoses of the 86 patients and method of diagnoses.

\begin{tabular}{|c|c|c|c|}
\hline Final diagnosis & $O L B$ & $T B B$ & $\begin{array}{l}\text { Clinical diagnosis (with } \\
\text { or without follow up) }\end{array}$ \\
\hline \multicolumn{4}{|l|}{ Cryptogenic fibrosing } \\
\hline alveolitis & 32 & & 9 \\
\hline Sarcoidosis & & 4 & 3 \\
\hline Histiocytosis X & 3 & & 2 \\
\hline Lymphangioleiomyomatosis & 3 & & \\
\hline \multicolumn{4}{|l|}{ Extrinsic allergic } \\
\hline alveolitis & 1 & & 7 \\
\hline $\begin{array}{l}\text { Lymphoma/lymphomatoid } \\
\text { granulomatosis/Wegener's }\end{array}$ & 3 & & \\
\hline Organising pneumonia & 2 & 2 & 1 \\
\hline Obliterative bronchiolitis & & & 1 \\
\hline $\begin{array}{l}\text { Bronchioloalveolar cell } \\
\text { carcinoma }\end{array}$ & & & $2^{\star}$ \\
\hline Pulmonary ossification & 1 & & 1 \\
\hline Silicosis & & & 1 \\
\hline Berylliosis & & 1 & \\
\hline Pulmonary haemosiderosis & 3 & & \\
\hline Drug induced alveolitis & 1 & & \\
\hline Pulmonary eosinophilia & & & 1 \\
\hline Lymphangitis carcinomatosa & & & 1 \\
\hline Alveolar proteinosis & & 1 & \\
\hline
\end{tabular}

OLB—open lung biopsy; TBB—-transbronchial biopsy.

${ }^{\star}$ Both diagnosed on percutaneous needle biopsy.
Israel) or an Imatron C-100 ultrafast electron beam CT scanner (Imatron, San Francisco, California); $3 \mathrm{~mm}$ sections were taken at $10 \mathrm{~mm}$ intervals and reconstructed with a high resolution algorithm. A limited number of prone scans were obtained in some patients. These are necessary to clarify gravitational effects which produce an increase in parenchymal opacification in the dependent part of the lungs on supine scan and may mimic the CT appearances of fibrosing alveolitis.

Two observers (DMH and MBR, consultant radiologists with a specialist interest in chest radiology) reviewed the chest radiographs and CT scans independently and on different occasions. The observers were asked to state whether or not the diagnosis was fibrosing alveolitis; if fibrosing alveolitis was considered to be the diagnosis they were asked to assign a degree of confidence: 1 for definite, 2 for probable, and 3 for unlikely. If fibrosing alveolitis was thought not to be the diagnosis this was recorded (a degree of confidence was not assigned for this category). No clinical details were provided and apart from being told that asbestos related disease was not included no information was provided regarding the range of diseases included in this series.

\section{DATA ANALYSIS}

Conventional techniques for evaluating diagnostic tests were applied: (a) sensitivity-the proportion of true positives correctly identified by the test (true positives/true positives + false negatives); (b) specificity-the proportion of true negatives correctly identified by the test (true negatives/true negatives + false positives); and (c) accuracy-the proportion of observations which are true positives and true negatives (true positives + true negatives/all observations). The results of the two observers were added. Results were treated in a dichotomous fashion. Diagnoses of fibrosing alveolitis on CT and chest radiography which were assigned grades 1 and 2 (definite and probable) were grouped and classified as positive diagnoses. Grade 3 (unlikely) diagnoses which would be considered indeterminate in clinical practice were classified together with the negative group.

The sensitivity, specificity, and accuracy of typical appearances on CT and chest radiography were calculated. Comparisons between CT and chest radiography were performed with McNemar's $\chi^{2}$ test (which confines analysis to cases in which tests give divergent results).

Results

The study group included 41 patients with fibrosing alveolitis and 45 patients with 17 different diffuse lung conditions (table 1).

On chest radiography the two observers positively and correctly diagnosed fibrosing alveolitis in 58 of $82(71 \%)$ observations. This increased to 73 of $82(89 \%)$ on high 
Table 2 Combined results of the two observers for sensitivity, specificity and accuracy on chest radiography and computed tomography (CT).

\begin{tabular}{lllll}
\hline & $\begin{array}{l}\text { Grade 1 } \\
\text { (definite) }\end{array}$ & $\begin{array}{l}\text { Grade 2 } \\
\text { (probable) }\end{array}$ & $\begin{array}{l}\text { Grade 3 } \\
\text { (unlikely) }\end{array}$ & $\begin{array}{l}\text { Not } \\
\text { fibrosing } \\
\text { alveolitis }\end{array}$ \\
\hline $\begin{array}{l}\text { Fibrosing alveolitis } \\
\quad \text { (41 patients, }\end{array}$ & & & & \\
$\quad$ 82 observations) & 24 & 34 & 13 & 11 \\
Chest radiography & 49 & 24 & 8 & 1 \\
CT & & & & \\
Non-fibrosing alveolitis & & & & \\
$\quad$ (45 patients, 90 & & & & \\
$\quad$ observations) & 4 & 13 & 29 & 54 \\
Chest radiography & 2 & 10 & 21 & 57 \\
\hline
\end{tabular}

Sensitivity for chest radiography, 58 of $82(71 \%)$; CT, 73 of $82(89 \%)$.

Specificity for chest radiography, 73 of 90 (81\%); CT, 78 of $90(87 \%)$.

Accuracy for chest radiography, 131 of $172(76 \%)$; CT, 151 of $172(88 \%)$

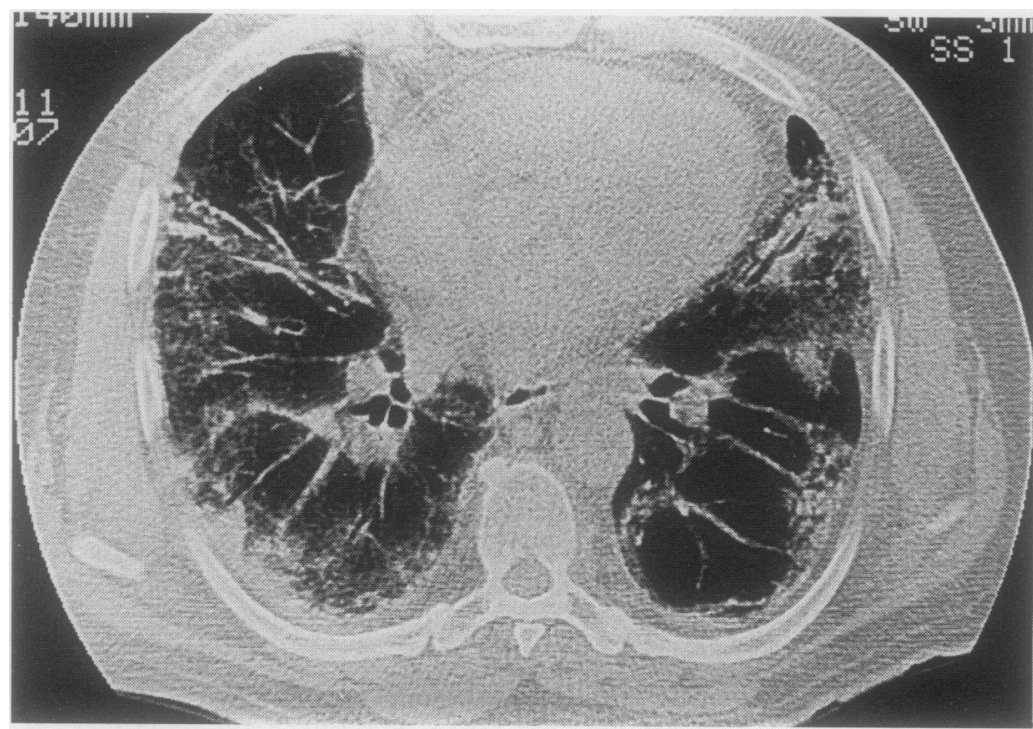

Figure 2 Dense peripheral consolidation in sarcoidosis; the subpleural distribution of disease is similar to fibrosing alveolitis. Several linear opacities represent areas of subsegmental collapse.

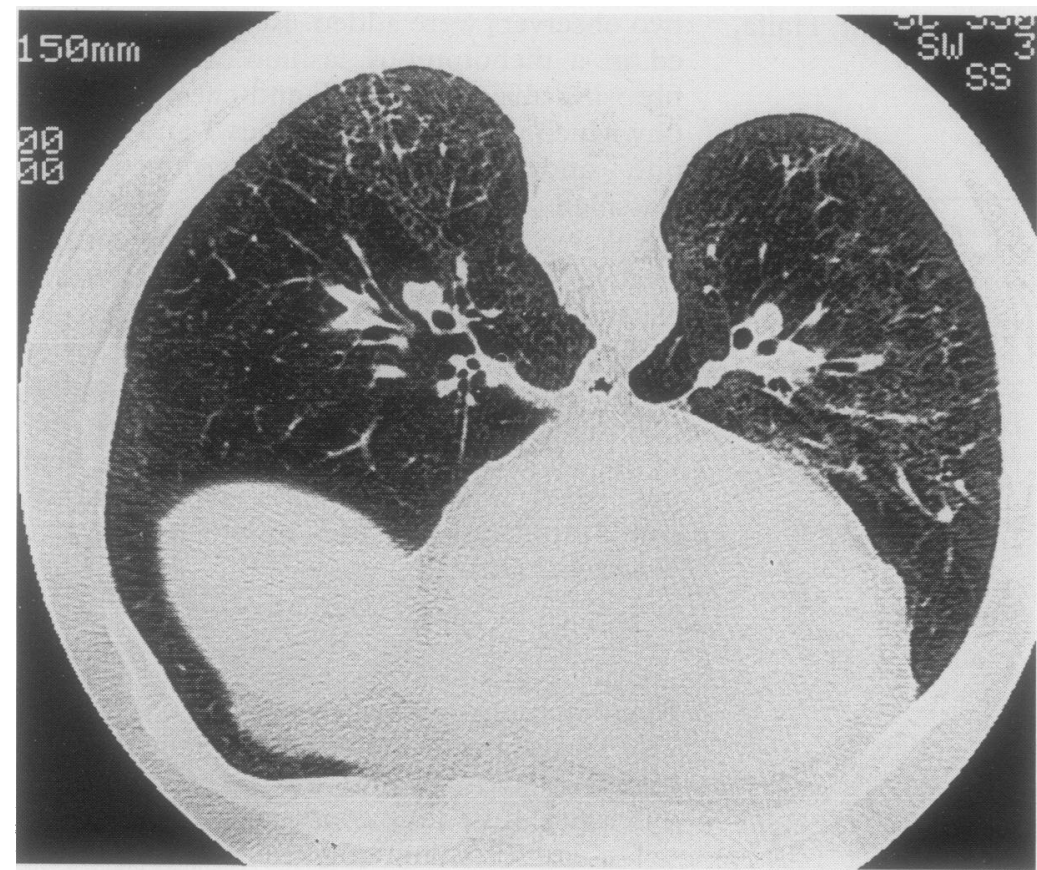

Figure 3 A reticular pattern in the posterobasal segments of the lower lobes in a patient with extrinsic allergic alveolitis; this pattern resembles that seen in fibrosing alveolitis (patient prone). resolution CT scans. A higher proportion of the correct positive diagnoses on CT scans were in the definite (grade 1) category (67\%) than were the diagnoses on chest radiography $(41 \%)$. Fibrosing alveolitis was incorrectly thought not to be the diagnosis (false negative diagnoses) in 24 of $82(29 \%)$ on chest radiographs and 9 of $82(11 \%)$ on CT.

On chest radiography a confident but incorrect (false positive) diagnosis of fibrosing alveolitis was made in 17 of $90(19 \%)$ observations; this reduced to 12 of $90(13 \%)$ on CT. Of the latter group only two diagnoses of fibrosing alveolitis were given by the observers as "definite", the remaining 10 were "probable" diagnoses.

To determine the clinical utility of predicting the diagnosis of fibrosing alveolitis from the typical CT appearances, sensitivity and specificity levels for chest radiography and CT were calculated. The sensitivity, specificity, and accuracy of CT was superior to chest radiography: CT had a sensitivity of $89 \%$, a specificity of $87 \%$ and an accuracy of $88 \%$ when a typical appearance (grades 1 or 2) was the criterion for making the diagnosis of fibrosing alveolitis. In contrast, chest radiography had a sensitivity of $71 \%$, a specificity of $81 \%$ and an accuracy of $76 \%$. The results are summarised in table 2 .

Overall CT was more accurate than chest radiography in correctly discriminating between fibrosing alveolitis and other interstitial lung diseases, ( $p<0.002$, McNemar's $\chi^{2}$ test). This difference was largely attributable to the superior sensitivity of the typical CT appearances $(p<0.05)$. The higher specificity of CT compared with chest radiography was not significant.

The conditions which were incorrectly diagnosed as fibrosing alveolitis on CT by the observers included extrinsic allergic alveolitis (five observations), sarcoidosis (two observations), cryptogenic organising pneumonia (two observations), pulmonary eosinophilia (two observations), and berylliosis (one observation). These incorrect diagnoses were made by one but not both observers in six cases (three extrinsic allergic alveolitis, two cryptogenic organising pneumonia, one berylliosis). In three cases (one each of sarcoidosis, extrinsic allergic alveolitis, and pulmonary eosinophilia) both observers (six observations) agreed but incorrectly diagnosed fibrosing alveolitis on CT. The abnormalities in these three cases had a striking peripheral subpleural distribution. One patient with sarcoidosis had evidence of dense peripheral consolidation on the CT scan (fig 2) and, unusually for fibrosing alveolitis, the extreme left lung base was spared. The chest radiograph in this case was felt by both observers to be inconsistent with a diagnosis of fibrosing alveolitis. In the patient with extrinsic allergic alveolitis misdiagnosed as probable fibrosing alveolitis (fig 3) closer critical examination showed similar sparing of the extreme lung bases. The patient with pulmonary eosinophilia had patchy peripheral consolidation on CT. 


\section{Discussion}

Recent advances in CT technology have resulted in high resolution CT images which correlate closely with the macroscopic appearances of pathological specimens. ${ }^{112}$ The high resolution CT appearances of many diffuse lung conditions including fibrosing alveolitis have now been described and they are often sufficiently characteristic to allow a specific diagnosis. ${ }^{13-15}$ The improvement in diagnostic capabilities of high resolution CT has led to a review of conventional clinical practice, particularly the need to confirm the diagnosis by open lung biopsy. ${ }^{16}$ Cryptogenic fibrosing alveolitis is a disease in which open lung biopsy is still often considered to be the final diagnostic arbiter. A firm diagnosis can be established by histological examination and, furthermore, the appearances of the biopsy specimens correlate with disease activity, prognosis, and response to therapy. ${ }^{1317} \mathrm{~A}$ more cellular histological appearance correlates with greater response to treatment and a longer mean survival time than a pattern of established fibrosis. Open lung biopsy, however, is an invasive procedure and complications can occur: in one series there was a major complication rate of $2.5 \%$ and minor complications in a further $2 \cdot 5 \% .{ }^{18}$ Moreover many patients with interstitial lung disease are too compromised to be considered for open lung biopsy. A further problem is that although biopsy specimens may be taken from more than one site, the method is open to sampling error. Furthermore, because of the geographical variation of the disease, specimens may not always reflect the spectrum of disease activity. By contrast high resolution CT samples a larger volume of lung and the apparent accuracy of high resolution CT in prediciting the diagnosis of fibrosing alveolitis in our study and others calls into question the traditional view that open lung biopsy is the gold standard in this context.

The results from our study show that for fibrosing alveolitis high resolution CT is clearly superior to conventional chest radiography with a confident correct diagnostic rate improving from $71 \%$ to $89 \%$. Similarly the false negative rate is reduced from $29 \%$ on chest radiography to $11 \%$ on high resolution CT. While the superior diagnostic accuracy of high resolution $\mathrm{CT}$ over chest radiography in many other diffuse lung diseases is well documented, it is notable that this has not been so clearcut in studies which have included small numbers of patients with fibrosing alveolitis: two studies ${ }^{15} 19$ showed no significant improvement in accuracy of high resolution CT over chest radiography in the diagnosis of fibrosing alveolitis. In another study which compared the accuracy of chest radiography and CT a confident diagnosis of fibrosing alveolitis improved from $87 \%$ on chest radiography to $95 \%$ on CT. ${ }^{14}$ Our study confirms the superior accuracy of CT over chest radiography in making a confident diagnosis of fibrosing alveolitis. Despite recent advances in the technique of chest radiology, notably the development of scanning equalisation radiography, there is no evidence that in the context of diffuse lung disease diagnostic accuracy is significantly improved with this technique. ${ }^{20}$

In our study the false positive diagnostic rate for fibrosing alveolitis decreased from $19 \%$ on chest radiography to $13 \%$ on CT. To determine how pathognomonic the radiographic appearances were in isolation the two observers were not provided with any clinical data. Furthermore the chest radiographs and scans were viewed independently of each other. In a clinical setting we believe the false positive diagnostic rate would be even lower when both investigations are viewed in conjunction and where there is access to full clinical information. It is also possible that our study design, with the question specifically directed towards a diagnosis of fibrosing alveolitis, may have introduced a bias towards a higher false positive rate. Nevertheless this study highlights those conditions which very rarely mimic fibrosing alveolitis on CT and which should be considered in the differential diagnosis. Sarcoidosis may occasionally appear as areas of peripheral consolidation ${ }^{21}$ and this may be confused with a subpleural distribution of very cellular fibrosing alveolitis as in one of our cases (fig 2). While the pulmonary fibrosis associated with chronic and repeated antigen exposure in extrinsic allergic alveolitis is almost invariably upper lobe in distribution, it may very rarely have an atypical distribution (fig 3). In practice patients with features of interstitial fibrosis, whatever the radiological distribution, should have a careful history taken for possible allergen exposure and serological evidence should also be taken into account. Cryptogenic organising pneumonia or bronchiolitis obliterans organising pneumonia may show a similar distribution to fibrosing alveolitis on CT. ${ }^{22}$ The pattern in cryptogenic organising pneumonia, however, is usually of multifocal, patchy areas of dense peripheral consolidation in contrast to the more homogeneous and less dense ground glass opacification seen in cellular fibrosing alveolitis. In pulmonary eosinophilia CT usually shows subsegmental and segmental or nodular areas of peripheral consolidation ${ }^{23}$ and typically there is an associated blood eosinophilia.

The range of diagnoses included in this study will not necessarily reflect the diagnoses encountered in everyday practice. With the exception of industrial lung diseases a wide variety of diverse conditions have been included in order to test the specificity of the typical CT appearances of fibrosing alveolitis. We acknowledge that the results reflect the experience of specialist radiologists practising in a tertiary referral centre. The study draws attention to those conditions which very rarely may mimic the appearances of fibrosing alveolitis on CT and we believe that an awareness of these possibilities should increase the diagnostic accuracy of CT in fibrosing alveolitis.

In conclusion our study shows that high 
resolution CT is superior to chest radiography in correctly discriminating between fibrosing alveolitis and other diffuse lung diseases. The need for an open lung biopsy to confirm the diagnosis in patients with suspected fibrosing alveolitis needs to be carefully considered.

1 Turner-Warwick M, Burrows B, Johnson A. Cryptogenic fibrosing alveolitis: clinical features and their influence on survival. Thorax 1980;35:171-80.

2 Jackson LK. Idiopathic pulmonary fibrosis. Clin Chest Med 1982;3:579-92.

3 Wright PH, Heard BE, Steel SJ, Turner-Warwick $M$. Cryptogenic fibrosing alveolitis: assessment by graded trephine lung biopsy histology compared with clinical, radiographic and physiological features. $\mathrm{Br} \mathcal{F}$ Dis Chest 1981;75:61-70.

4 King TE. Diagnostic advances in idiopathic pulmonary fibrosis. Chest 1991;100:238-41.

5 Fraser RG, Paré JAP, Paré PD, Fraser RS, Genereux GP. Pulmonary disease of unknown origin. In: Diagnosis of diseases of the chest. 3rd edn. Philadelphia: Saunders, 1991:2662-4.

6 Wilson AG. Immunologic diseases of the lungs. In: Armstrong P, Wilson AG, Dee P, eds. Imaging of diseases of the chest. Chicago: Year Book, 1990:464-72.

7 Epler GR, McLoud TC, Gaensler EA, Mikus JP, Carrington CB. Normal chest roentgenograms in chronic diffuse infiltrative lung disease. $N \mathrm{Engl} f \mathrm{Med}$ 1978;298:935-9.

8 Müller NL, Miller RR, Webb WR, Evans KG, Ostrow DN. Fibrosing alveolitis: CT pathologic correlation. Radiology 1986;160:585-8.

9 Strickland B, Strickland NH. The value of high definition, narrow section computed tomography in fibrosing alveolitis. Clin Radiol 1988;39:589-94.

10 Müller NL, Miller RR. Computed tomography of chronic diffuse infiltrative lung disease (part 1). Am Rev Respir Dis 1990;142:1206-15.
11 Hruban RH, Meziane MA, Zerhouni EA, Khouri NF Fishman EK, Wheeler PS, et al. High resolution computed tomography of inflation-fixed lungs. Am Rev Respir Dis 1987;136:935-40.

12 Webb WR, Stein MG, Finkbeiner WE, Im JG, Lynch D, Gamsu G. Normal and diseased isolated lungs: high resolution CT. Radiology 1988;166:81-7.

13 Bergin CJ, Coblentz CL, Chiles C, Bell DY, Castellino RA. Chronic lung diseases: specific diagnosis by using CT. $A$ FR 1989;152:1183-8

14 Mathieson JR, Mayo JR, Staples CA, Müller NL. Chronic diffuse infiltrative lung disease: comparison of diagnostic accuracy of CT and chest radiography. Radiology 1989;171:111-6.

15 Padley SPG, Hansell DM, Flower CDR, Jennings $P$. Comparative accuracy of high resolution computed tomography and chest radiography in the diagnosis of chronic diffuse infiltrative lung disease. Clin Radiol 1991;44:227-31.

16 Müller NL Clinical value of high resolution CT in chronic diffuse lung disease. AfR 1992;157:1163-70

17 Carrington CB, Gaensler EA, Coutu RE, Fitzgerald MX Gupta RG. Natural history and treated course of usual and desquamative interstitial pneumonia. $N \mathrm{Engl} f \mathrm{Med}$ 1978;298:801-9.

18 Gaensler EA, Carrington CB. Open lung biopsy for chronic diffuse infiltrative lung disease: clinical, roentgenographic and physiological correlations in 502 roentgenographic and physiological correlatic
patients. Ann Thorac Surg 1980;30:411-26.

19 Grenier P, Valeyre D, Cluzel P, Brauner MW, Lenoir S, Chastang $\mathrm{C}$. Chronic diffuse interstitial lung disease: diagnostic value of chest radiography and high resolution CT. Radiology 1991;179:123-32.

20 Hansell DM, Coleman R, du Bois RM, Carr DH, Goodman LR, Kerr IH, et al. Advanced multiple beam equalization radiography (AMBER) in the detection of diffuse lung disease. Clin Radiol 1991;44:227-31.

21 Glazer HS, Levitt RG, Shackleford GD. Peripheral pulmonary infiltrates in sarcoidosis. Chest 1984;86:741-4.

22 Müller NL, Staples CA, Miller RR. Bronchiolitis obliterans organizing pneumonia: CT features in 14 patients. ans organizing pneum

23 Mayo JR, Müller NL, Road J, Sisler J, Lillington G Chronic eosinophilic pneumonia: CT findings in six cases. AfR 1989;153:727-30. 